\title{
Dual-Species Plasmas Illustrate MHD Flows
}

\author{
Eve V. Stenson and Paul M. Bellan
}

\begin{abstract}
Plasma loops created in the laboratory strongly resemble structures observed in the solar corona. For example, both solar coronal loops and experimental loops exhibit remarkably uniform axial cross sections. A magnetohydrodynamic theory that was proposed to explain this phenomenon predicts that a plasma loop whose axial magnetic field is constricted at both footpoints will experience bulk flows into the loop from both ends. To test this theory, dual-species plasma loops were formed by supplying a different neutral gas to each of the two footpoints. Optical filters were then used to separately image the motion of different sections of the plasma. Bulk flows were, in fact, observed.
\end{abstract}

Index Terms-Flows, laboratory solar physics, loops, magnetohydrodynamic (MHD).

$\mathbf{C}$ URRENTS beneath the surface of the sun produce arched magnetic fields extending out into the corona [1]. A similar arched field is created in the laboratory by coils located behind the electrodes of a magnetized plasma gun (Fig. 1). The vacuum magnetic field generated by such processes diminishes with increasing distance from the source, causing the field lines to spread out. Hence, one would expect plasma loops in these environments to be much wider at their apex than at their footpoints. This is not the case though. Coronal loops exhibit little axial variation, being only $30 \%$ larger in diameter at the apex than at the footpoints [2]. Similar axial uniformity is observed in laboratory plasma loops [3].

A model has been proposed to explain how current-carrying magnetic flux tubes in the ideal magnetohydrodynamic (MHD) regime might achieve this uniformity [4]. This model predicts that, if a flux tube were bulged at its axial midpoint, JxB forces would produce axial flows from the narrower regions to the wider ones. These bulk flows would carry frozen-in azimuthal flux. The "piling up" of azimuthal flux would cause the bulge to pinch down at the axial midpoint, decreasing the axial variation.

The axial magnetic field in a laboratory plasma loop is constricted at both footpoints, each of which is located on an electrode (Fig. 1). Hence, two flows would be expected: one coming from the anode (parallel to the current) and one coming from the cathode (antiparallel to the current). Spectroscopic measurements of laboratory plasma loops have shown blue shifts, indicating the presence of such flows [5]. To further test the theory proposed in [4], experiments were performed using two different species for the neutral gas from which the plasma

Manuscript received December 7, 2007; revised April 1, 2008. This work was supported in part by the U.S. Department of Energy and in part by the Air Force Office of Scientific Research.

The authors are with the California Institute of Technology, Pasadena, CA 91125 USA (e-mail: eve@ caltech.edu).

Digital Object Identifier 10.1109/TPS.2008.927095

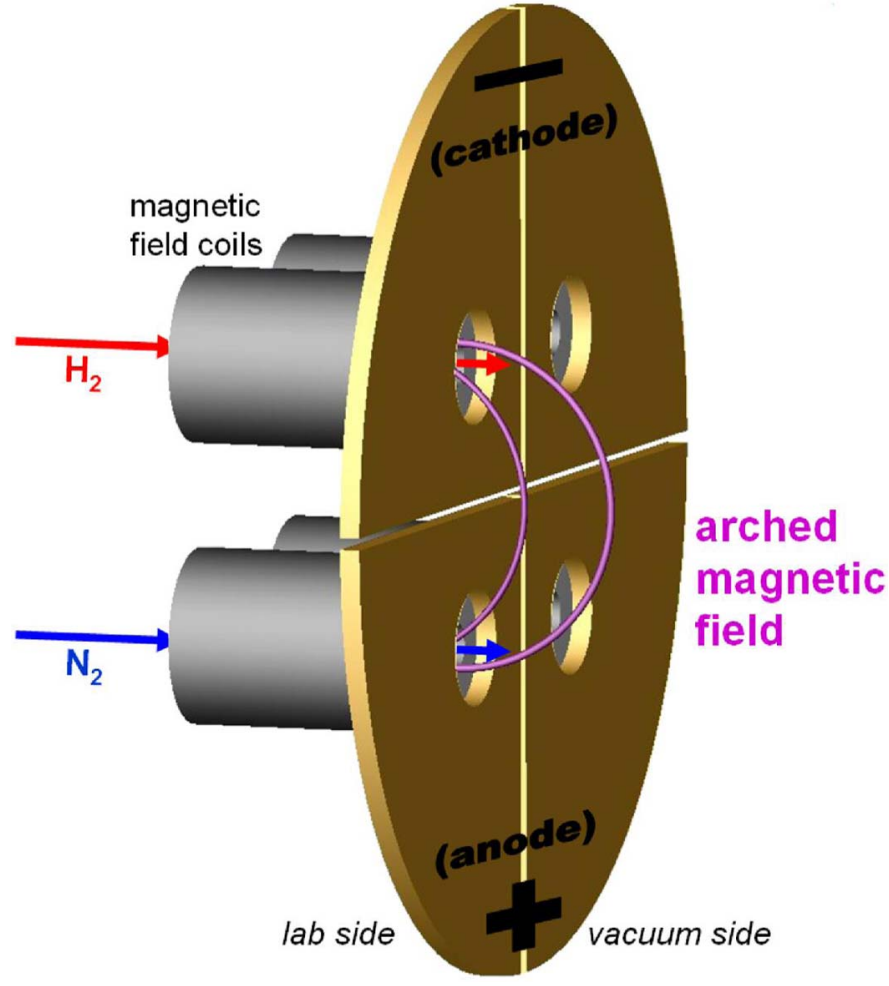

Fig. 1. Experimental configuration. The coil current is constant on the time scale of the plasma. As gas enters the chamber, 3-6 kV is applied across the electrodes, ionizing the gas and driving a current of $\sim 40 \mathrm{kA}$ through the plasma, from anode to cathode. (The nozzles and coils located on the far side of the electrode were turned off for this experiment.)

is formed. A different gas was supplied to each of the two footpoints of the loop.

Prior to each plasma shot, the vacuum chamber is pumped down to $\sim 5 \times 10^{-7}$ torr. A magnetic field of $0.1-0.3 \mathrm{~T}$ is established by applying a voltage pulse to coils located behind the electrodes, each coaxial with a gas nozzle (Fig. 1). This field lasts on the order of milliseconds, making it constant on the time scale of the plasma, which has a duration of $\sim 10 \mu \mathrm{s}$. Next, fast gas valves are triggered, allowing a small amount of neutral gas to enter the chamber. As the gas is released, $3-6 \mathrm{kV}$ is applied across the electrodes. This ionizes the gas and then drives a current of $40-80 \mathrm{kA}$ through the resulting plasma loop. Typical densities are in the range of $10^{20}-10^{21} \mathrm{~m}^{-3}$, and temperatures are in the range of $2-5 \mathrm{eV}$.

The plasma is imaged by an Imacon 200 (DRS Technologies) gated intensified charge-coupled-device camera. Up to 16 frames can be recorded per shot. Exposures are typically in the range of 20-50 ns, and interframe times are in the range of $100-500 \mathrm{~ns}$. In some shots, optical filters are used to selectively 


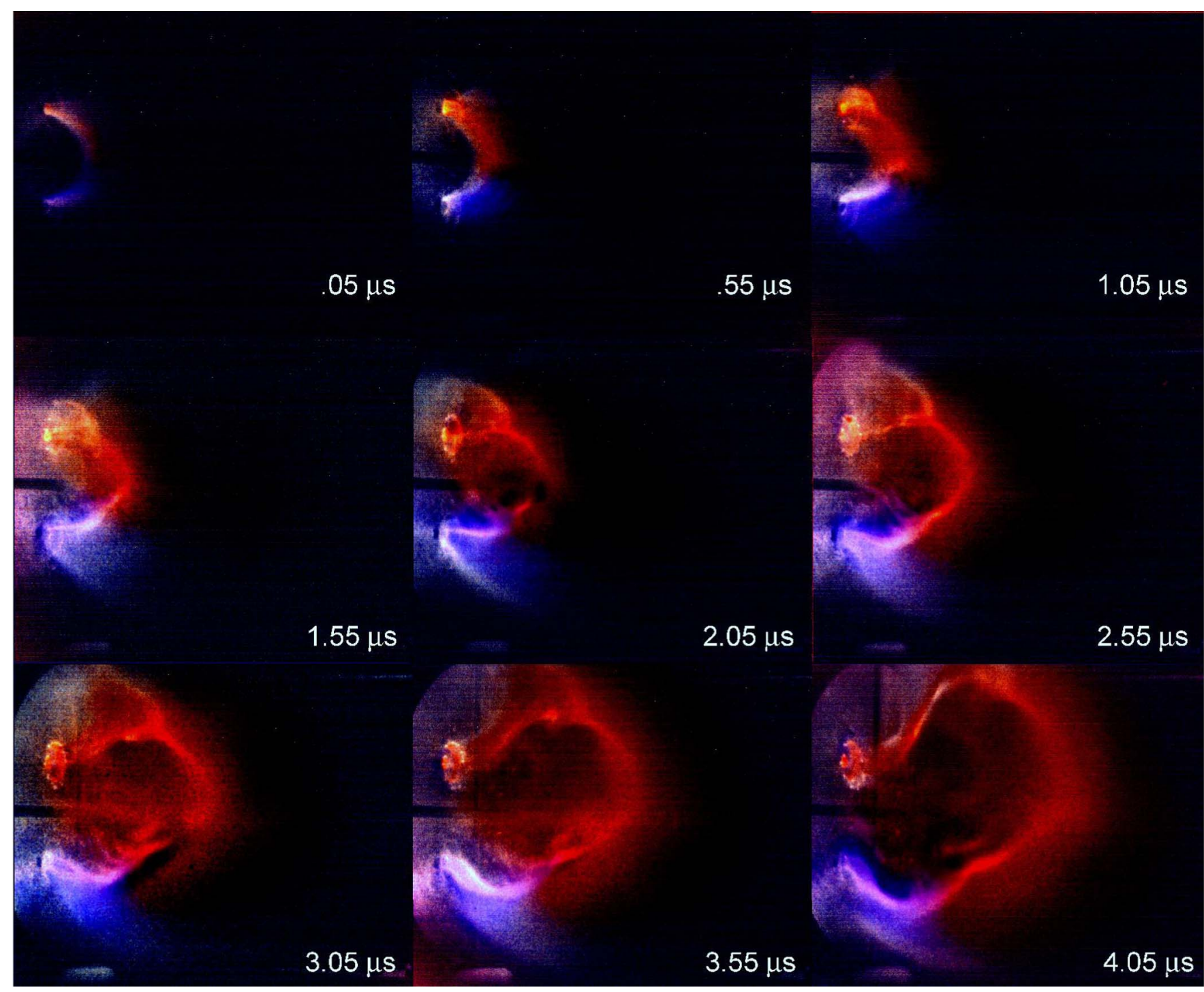

Fig. 2. Dual-species plasma is formed when hydrogen is supplied from a nozzle in the cathode (top electrode) of a magnetized plasma gun and nitrogen is supplied from a nozzle in the anode (bottom electrode). MHD forces generate bulk flows into the loop from both footpoints. Sections of the plasma emitting 656-nm light (H-alpha) are colored red, and sections of the plasma emitting 402-nm light (various ionized $\mathrm{N}$ lines) are colored blue.

image a particular emission wavelength. For example, a 656-nm filter is used to image $\mathrm{H}$-alpha emissions, and a 402-nm filter is used to image emission wavelengths from singly or doubly ionized nitrogen atoms. Due to the high repeatability of the experiments, images of multiple shots can then be colored and digitally overlaid to produce color visualizations of the plasma. In Fig. 2, three shots were used. An unfiltered shot was overlaid with both a shot imaged with the 402-nm filter (colored blue) and a shot imaged with the 656-nm filter (colored red).

In the resulting images, two flows can be identified: one originating from each electrode and, hence, one of each gas. Flow speeds, which are calculated by image tracking, depend on mass density; they are faster for lighter gases than for heavier gases. They do not depend on the direction of the bias vacuum field; reversing the direction leaves the flows unchanged. The speed of each flow is also independent of whether it originates from the cathode or the anode; this is seen by switching which gas is supplied to which electrode.
Thus, the flows from the footpoints predicted by the model proposed in [4] have clearly been observed. Because this is a general MHD mechanism, these results imply that similar phenomena should also be present in the solar corona and, indeed, in all current-carrying magnetic flux tubes.

\section{REFERENCES}

[1] M. J. Aschwanden, Physics of the Solar Corona. Chichester, U.K.: Springer-Verlag, 2006, pp. 16-17.

[2] J. A. Klimchuk, "Cross-sectional properties of coronal loops," Sol. Phys., vol. 193 , no. $1 / 2$, pp. $53-75$, Apr. 2000.

[3] J. H. Hansen and P. M. Bellan, "Experimental demonstration of how strapping fields can inhibit solar prominence eruptions," Astrophys. J., vol. 563, no. 2, pp. L183-L186, Dec. 2001.

[4] P. M. Bellan, "Why current-carrying magnetic flux tubes gobble up plasma and become thin as a result," Phys. Plasmas, vol. 10, no. 5, pp. 1999-2008, May 2003.

[5] S. K. P. Tripathi, P. M. Bellan, and G. S. Yun, "Observation of kinetic plasma jets in a coronal-loop simulation experiment," Phys. Rev. Lett., vol. 98 , no. 13, p. 135002 , Mar. 2007 